PROCEEDINGS OF THE

AMERICAN MATHEMATICAL SOCIETY

Volume 138, Number 4, April 2010, Pages 1317-1331

S 0002-9939(09)10152-1

Article electronically published on December 8, 2009

\title{
DISCRETE PAINLEVÉ EQUATIONS FOR RECURRENCE COEFFICIENTS OF SEMICLASSICAL LAGUERRE POLYNOMIALS
}

\author{
LIES BOELEN AND WALTER VAN ASSCHE \\ (Communicated by Peter A. Clarkson)
}

\begin{abstract}
We consider two semiclassical extensions of the Laguerre weight and their associated sets of orthogonal polynomials. These polynomials satisfy a three-term recurrence relation. We show that the coefficients appearing in this relation satisfy discrete Painlevé equations.
\end{abstract}

\section{INTRODUCTION}

Discrete Painlevé equations ( $\mathrm{dP}$ ) are second-order, nonlinear difference equations which have a continuous Painlevé equation as a continuous limit. They pass an integrability test called singularity confinement 9 . This integrability detector is the discrete analogue of the Painlevé property for differential equations. The discrete Painlevé equations share many features of their continuous counterparts: some can be fitted into degeneration cascades; for others Lax pairs have been established, hierarchies have been computed, and special solutions can be found, as well as Miura and Bäcklund transformations. At least one interesting difference between discrete and continuous Painlevé is the following: there are a lot more dP's than the six continuous ones (and as we name the dP's after their continuous limit, there are various nonequivalent $\mathrm{dP}_{\mathrm{I}}$ 's, etc.). There is a 'standard list' ([7]) consisting of the earliest derived discrete Painlevé equations. A more elaborate list was compiled by Peter Clarkson and can be found in [14]. For a comprehensive overview of discrete Painlevé equations, see [8].

Orthonormal polynomials on the real line obey a three-term recurrence relation of the form

$$
x p_{n}(x)=a_{n+1} p_{n+1}(x)+b_{n} p_{n}(x)+a_{n} p_{n-1}(x)
$$

(with $p_{-1}=0$ ). The recurrence coefficients of classical orthogonal polynomials can be obtained explicitly. For semiclassical weights, i.e., weights $w$ satisfying a Pearson equation $D(\sigma w)=\tau w$, where $\sigma$ and $\tau$ are polynomials with $\operatorname{deg} \sigma>2$ or $\operatorname{deg} \tau \neq 1$, the recurrence coefficients obey nonlinear recurrence relations, which in many cases can be identified as discrete Painlevé equations (4 , [5], [11, [12, [14]). Our aim is to find the nonlinear recurrence relations explicitly for other semiclassical

Received by the editors February 23, 2009, and, in revised form, July 2, 2009.

2010 Mathematics Subject Classification. Primary 39A13, 33C45; Secondary 42C05.

Key words and phrases. Discrete Painlevé equations, orthogonal polynomials.

This research was supported by K. U. Leuven Research Grant OT/08/033, FWO Research Grant G.0427.09 and the Belgian Interuniversity Attraction Poles Programme P6/02. 
weights and to identify them as discrete Painlevé equations. In this way we hope to establish a connection between semiclassical orthogonal polynomials and discrete Painlevé equations. The fact that one deals with discrete Painlevé equations for the recurrence coefficients indicates that no simple formula will be available for the recurrence coefficients (except for some special cases that can be identified). On the other hand, the integrability of the Painlevé equations implies that the complexity of the recurrence coefficients does not grow exponentially [1. Painlevé equations are on the borderline between linearisability and nonintegrability 8 .

In this paper we make the connection between orthogonal polynomials which are semiclassical extensions of the Laguerre polynomials and some discrete Painlevé equations. We prove the following results:

Theorem 1.1. Consider the orthonormal polynomials with respect to the weight

$$
w(x)=x^{\alpha} e^{-x^{2}+t x}, \quad x>0,
$$

with $\alpha>-1$ and $t \in \mathbb{R}$. The recurrence coefficients in the three-term recurrence relation

$$
x p_{n}(x)=a_{n+1} p_{n+1}(x)+b_{n} p_{n}(x)+a_{n} p_{n-1}(x)
$$

satisfy

$$
\left\{\begin{aligned}
x_{n} x_{n-1} & =\frac{y_{n}+z_{n}}{y_{n}^{2}-\frac{\alpha^{2}}{4}} \\
y_{n}+y_{n+1} & =\frac{1}{x_{n}}\left(\frac{t}{\sqrt{2}}-\frac{1}{x_{n}}\right),
\end{aligned}\right.
$$

where $z_{n}=n+\alpha / 2, y_{n}=2 a_{n}^{2}-n-\alpha / 2$ and $x_{n}=\sqrt{2} /\left(t-2 b_{n}\right)$.

The equations in (1.1) bear similarities with an asymmetric Painlevé equation $\mathrm{dP}_{\mathrm{IV}}$ in the list of Clarkson [14],

$$
\left\{\begin{aligned}
u_{n} u_{n-1} & =\frac{a\left(v_{n}+z_{n}-b\right)}{v_{n}^{2}-\gamma^{2}}, \\
v_{n}+v_{n+1} & =\frac{c}{u_{n}}+\frac{z_{n+1 / 2}+d}{u_{n}-1}
\end{aligned}\right.
$$

with $z_{n}=\alpha_{1} n+\beta_{1}$. In fact, we can obtain (1.1) from (1.2) by putting $u_{n}=x_{n} / \epsilon$, $v_{n}=\epsilon y_{n}, z_{n}=\epsilon \alpha n+\epsilon \beta, a=1 / \epsilon, \gamma=\epsilon \alpha / 2, c=1 / \epsilon+t / \sqrt{2}, d=-1 / \epsilon$ and letting $\epsilon \rightarrow 0$. It was identified as a Miura transform of an asymmetric $\mathrm{dP}_{\mathrm{I}}$ in [13], a result we will reestablish from the point of view of orthogonal polynomials in Section 2.3 .

The case $t=0$ gives a somewhat simpler equation:

Corollary 1.2. If we consider the orthonormal polynomials with weight

$$
w(x)=x^{\alpha} e^{-x^{2}}, \quad x>0,
$$

then the recurrence coefficients satisfy

$$
\left(y_{n}+y_{n+1}\right)\left(y_{n}+y_{n-1}\right)=\frac{\left(y_{n}^{2}-\alpha^{2} / 4\right)^{2}}{\left(y_{n}+z_{n}\right)^{2}},
$$

where $z_{n}=n+\alpha / 2$ and $y_{n}=2 a_{n}^{2}-n-\alpha / 2$. The recurrence coefficients $b_{n}$ can be obtained from

$$
2 b_{n}^{2}=-y_{n}-y_{n+1} \text {. }
$$


Equation (1.3) is an instance of $\mathrm{dP}_{\mathrm{IV}}$ which was found in [6]. Theorem 1.1 and Corollary 1.2 will both be proven in Section 2. In Section 3 we prove

Theorem 1.3. Consider the monic orthogonal polynomials with respect to the weight

$$
w(x)=\frac{x^{\alpha}}{\left(-x^{2} ; q^{2}\right)_{\infty}}, \quad x>0,
$$

with $\alpha>0$ and $0<q<1$. The recurrence coefficients in the three-term recurrence relation

$$
x P_{n}(x)=P_{n+1}(x)+\alpha_{n} P_{n}(x)+\beta_{n} P_{n-1}(x)
$$

satisfy

$$
\left(x_{n} x_{n+1}-1\right)\left(x_{n} x_{n-1}-1\right)=\frac{q^{-2 n-\alpha}\left(x_{n}-q^{-\alpha / 2}\right)^{2}\left(x_{n}-q^{\alpha / 2}\right)^{2}}{\left(x_{n}-q^{-n-\alpha / 2}\right)^{2}},
$$

where $x_{n}=q^{-n-\alpha / 2}\left(1-q^{2 n+\alpha-1} \beta_{n}\right)$. We have $\alpha_{0}^{2}=x_{0} x_{1}+1$. The remaining recurrence coefficients $\alpha_{n}$ for $n \geq 1$ can be obtained from

$\alpha_{n}^{2} x_{n}^{2} q^{2 n+\alpha}=1+x_{n} x_{n+1}+\left(x_{n} x_{n-1}-1\right)\left(1-q^{n+\alpha / 2} x_{n}\right)^{2}+2 x_{n}\left(x_{n}-q^{\alpha / 2}-q^{-\alpha / 2}\right)$.

Equation (1.4) is an instance of the $q$-Painlevé V equation in the list of Clarkson [14; see also [8, p. 269].

\section{2. $\mathrm{dP}_{\text {IV }}$ FOR A SEMIClASSiCAL LAGUERRE WEIGHT}

We consider $w(x)=x^{\alpha} e^{-x^{2}+t x}$ on $\mathbb{R}^{+}$with $\alpha>-1$ and $t \in \mathbb{R}$. Denote the orthonormal polynomials with respect to this weight by $\left\{p_{n}\right\}$. The orthonormality condition is

$$
\int_{0}^{\infty} p_{n}(x) p_{m}(x) w(x) d x=\delta_{m n} .
$$

We will use the following notation for the coefficients of $p_{n}$ :

$$
p_{n}(x)=\gamma_{n} x^{n}+\delta_{n} x^{n-1}+\cdots,
$$

where $\gamma_{n}>0$. The three-term recurrence relation then takes the form

$$
x p_{n}(x)=a_{n+1} p_{n+1}(x)+b_{n} p_{n}(x)+a_{n} p_{n-1}(x)
$$

with

$$
\begin{gathered}
a_{n}=\int_{0}^{\infty} x p_{n}(x) p_{n-1}(x) w(x) d x, \\
b_{n}=\int_{0}^{\infty} x p_{n}^{2}(x) w(x) d x .
\end{gathered}
$$

It follows that all $b_{n}$ are positive. Comparing leading coefficients on both sides of this identity expresses $a_{n}$ as the ratio of the leading coefficients of the polynomials:

$$
a_{n}=\frac{\gamma_{n-1}}{\gamma_{n}}
$$

for $n>0$ and $a_{0}=0$. Hence all $a_{n}$ are positive for $n \geq 1$.

In Sections 2.1 and 2.2 we prove Theorem 1.1 and Corollary 1.2 We look at the relation between the recurrence coefficients of the orthogonal polynomials with respect to $w$ and those of the Freud weight in Section 2.3, recovering a result of [13. We conclude this section by looking at extensions of $w$ to the real line. 
2.1. Deriving the Lax pair. We rewrite the three-term recurrence relation in matrix form

$$
x P=J P
$$

with

$$
P=\left(\begin{array}{c}
p_{0} \\
p_{1} \\
p_{2} \\
\vdots
\end{array}\right), \quad J=\left(\begin{array}{ccccc}
b_{0} & a_{1} & 0 & 0 & \cdots \\
a_{1} & b_{1} & a_{2} & 0 & \cdots \\
0 & a_{2} & b_{2} & a_{3} & \\
\vdots & & \ddots & \ddots & \ddots
\end{array}\right)
$$

where $J$ is the three-diagonal Jacobi matrix. The Pearson equation for $w$ is

$$
[x w(x)]^{\prime}=\left(-2 x^{2}+t x+\alpha+1\right) w(x) .
$$

We consider the Fourier expansion of the polynomial $x p_{n}^{\prime}(x)=\sum_{k=0}^{n} \alpha_{k} p_{n-k}$ with

$$
\alpha_{k}=\int_{0}^{\infty} x p_{n}^{\prime}(x) p_{n-k}(x) w(x) d x .
$$

Integration by parts and the Pearson equation give

$\alpha_{k}=-\int_{0}^{\infty} p_{n}(x) x p_{n-k}^{\prime}(x) w(x) d x+\int_{0}^{\infty} p_{n}(x) p_{n-k}(x)\left(2 x^{2}-t x-\alpha-1\right) w(x) d x$.

These integrals are zero for $k>2$ due to orthonormality. For $k=2$ we have

$$
\alpha_{2}=2 \int_{0}^{\infty} p_{n}(x) x^{2} p_{n-2}(x) w(x) d x
$$

which is equal to $2 a_{n} a_{n-1}$ by applying the recurrence relation twice on $p_{n-2}$. Furthermore, by comparing the coefficients of $x^{n}$, we see that $\alpha_{0}=n$. In matricial form, we have

$$
x P^{\prime}=L P \quad \text { with } L=\left(\begin{array}{ccccc}
0 & 0 & 0 & 0 & \cdots \\
A_{1} & 1 & 0 & 0 & \cdots \\
2 a_{2} a_{1} & A_{2} & 2 & 0 & \cdots \\
0 & 2 a_{3} a_{2} & A_{3} & 3 & \\
\vdots & & \ddots & \ddots & \ddots
\end{array}\right)
$$

with

$$
A_{n}=\int_{0}^{\infty} x p_{n}^{\prime}(x) p_{n-1}(x) w(x) d x .
$$

Together with the recurrence relation, this leads to the following Lax pair:

$$
\left\{\begin{aligned}
x P & =J P \\
x P^{\prime} & =L P .
\end{aligned}\right.
$$

In the next section, we derive the compatibility condition for this Lax pair. From this condition we will be able to recover the recurrence coefficients as solutions of $\mathrm{dP}_{\mathrm{IV}}$. 
2.2. Computations. The compatibility condition of the Lax pair can be found by calculating $x(x P)^{\prime}$ in two ways and is

$$
J L-L J=J .
$$

This gives rise to three nontrivial equations:

$$
\begin{aligned}
b_{n} & =a_{n+1} A_{n+1}-a_{n} A_{n}, \\
A_{n}\left(b_{n-1}-b_{n}\right)+2 a_{n} & =2 a_{n}\left(a_{n+1}^{2}-a_{n-1}^{2}\right), \\
a_{n-1} A_{n}-a_{n} A_{n-1} & =2 a_{n} a_{n-1}\left(b_{n}-b_{n-2}\right) .
\end{aligned}
$$

Dividing (2.3) by $a_{n} a_{n-1}$ and taking a telescopic sum we get

$$
\frac{A_{n}}{a_{n}}=\frac{A_{1}}{a_{1}}+2\left(b_{n}+b_{n-1}-b_{1}-b_{0}\right)
$$

Observe that

$$
A_{1}=-\gamma_{0} \int_{0}^{\infty} p_{1}(x)\left[-2 x^{2}+t x+\alpha+1\right] w(x) d x .
$$

As the polynomial occurring in this integral has the Fourier expansion

$$
-2 x^{2}+t x+\alpha+1=\frac{-2}{\gamma_{2}} p_{2}(x)+\frac{1}{\gamma_{1}}\left(2 \frac{\delta_{2}}{\gamma_{2}}+t\right) p_{1}(x)+\text { constant },
$$

we find $A_{1}=a_{1}\left(2 b_{0}+2 b_{1}-t\right)$. Hence

$$
A_{n}=a_{n}\left(2 b_{n}+2 b_{n-1}-t\right) .
$$

Using this expression in (2.2) we obtain, after dividing by $a_{n}$ and taking a telescopic sum,

$$
2\left(a_{n}^{2}+b_{n}^{2}+a_{n+1}^{2}\right)-t b_{n}=2 n+2\left(a_{0}^{2}+b_{0}^{2}+a_{1}^{2}\right)-t b_{0} .
$$

One computes

$$
\begin{aligned}
2\left(a_{0}^{2}+b_{0}^{2}+a_{1}^{2}\right)-t b_{0} & =\int_{0}^{\infty}\left(2 x^{2}-t x\right) p_{0}^{2}(x) w(x) d x \\
& =-\gamma_{0}^{2} \int_{0}^{\infty}[x w(x)]^{\prime} d x+\alpha+1=\alpha+1
\end{aligned}
$$

hence

$$
2\left(a_{n}^{2}+b_{n}^{2}+a_{n+1}^{2}\right)=t b_{n}+2 n+\alpha+1 .
$$

Taking a telescopic sum of (2.1), we get

$$
a_{n} A_{n}=\sum_{j=0}^{n-1} b_{j} .
$$

Multiplying (2.2) by $a_{n}$ and taking a telescopic sum yields

$$
\begin{aligned}
2 a_{n}^{2} a_{n+1}^{2}-2 \sum_{j=0}^{n} a_{j}^{2} & =\sum_{j=1}^{n} a_{j} A_{j}\left(b_{j-1}-b_{j}\right) \\
& =\sum_{j=0}^{n-1}\left(a_{j+1} A_{j+1}-a_{j} A_{j}\right) b_{j}-a_{n} A_{n} b_{n} \\
& =\sum_{j=0}^{n-1} b_{j}^{2}-a_{n} A_{n} b_{n},
\end{aligned}
$$


where we have used (2.1) in the last line. We get, using (2.5) and (2.6),

$$
\begin{aligned}
2 a_{n}^{2} a_{n+1}^{2}+a_{n} A_{n} b_{n} & =\sum_{j=0}^{n-1}\left(a_{j}^{2}+b_{j}^{2}+a_{j+1}^{2}\right)+a_{n}^{2}=\frac{1}{2} \sum_{j=0}^{n-1}\left(t b_{j}+2 j+\alpha+1\right)+a_{n}^{2} \\
& =\frac{t}{2} a_{n} A_{n}+\frac{n(n+\alpha)}{2}+a_{n}^{2},
\end{aligned}
$$

or by using (2.4),

$$
4 a_{n}^{2} a_{n+1}^{2}=n(n+\alpha)+2 a_{n}^{2}-a_{n}^{2}\left(2 b_{n}-t\right)\left(2 b_{n}+2 b_{n-1}-t\right) .
$$

Now consider the case $t=0$. We use (2.5) to substitute $b_{n}^{2}$ in (2.7) and get

$$
4 a_{n}^{2} b_{n} b_{n-1}=\left(2 a_{n}^{2}-n\right)\left(2 a_{n}^{2}-n-\alpha\right) .
$$

Squaring this equation and substituting $b_{n}^{2}$ and $b_{n-1}^{2}$, using (2.5) we obtain $\left(2 a_{n}^{2}\right)^{2}\left(2 a_{n}^{2}+2 a_{n+1}^{2}-2 n-\alpha-1\right)\left(2 a_{n}^{2}+2 a_{n-1}^{2}-2 n+\alpha+1\right)=\left(2 a_{n}^{2}-n\right)^{2}\left(2 a_{n}^{2}-(n+\alpha)\right)^{2}$. Using the substitution $y_{n}=2 a_{n}^{2}-n-\alpha / 2$ gives

$$
\left(y_{n}+y_{n+1}\right)\left(y_{n}+y_{n-1}\right)=\frac{\left(y_{n}^{2}-\frac{\alpha^{2}}{4}\right)^{2}}{\left(y_{n}+z_{n}\right)^{2}}
$$

where $z_{n}=n+\alpha / 2$, thus proving Corollary 1.2. This is an instance of $\mathrm{dP}_{\mathrm{IV}}$, which was found in [6]. The first initial value, $y_{0}=-\alpha / 2$, follows from the fact that $a_{0}=0$. As

$$
a_{1}^{2}=\frac{\mu_{2} \mu_{0}-\mu_{1}^{2}}{\mu_{0}^{2}},
$$

where $\mu_{k}$ is the $k$ th moment of the weight $w$, we find for the second initial condition, $y_{1}=2 \frac{\mu_{2} \mu_{0}-\mu_{1}^{2}}{\mu_{o}^{2}}-1-\frac{\alpha}{2}$. Using integration by parts one finds

$$
\mu_{k}=\frac{2}{\alpha+k+1} \mu_{k+2}
$$

so we have

$$
y_{1}=\frac{\alpha}{2}-\frac{2 \mu_{1}^{2}}{\mu_{0}^{2}} .
$$

The case $t \neq 0$ doesn't allow a reduction of the system

$$
\left\{\begin{array}{l}
2 a_{n}^{2}+2 b_{n}^{2}+2 a_{n+1}^{2}=t b_{n}+2 n+\alpha+1, \\
4 a_{n}^{2} a_{n+1}^{2}=n(n+\alpha)+2 a_{n}^{2}-a_{n}^{2}\left(2 b_{n}-t\right)\left(2 b_{n}+2 b_{n-1}-t\right)
\end{array}\right.
$$

to a single equation. Using the substitutions $y_{n}=2 a_{n}^{2}-n-\alpha / 2$ and $x_{n}=\sqrt{2} \frac{1}{t-2 b_{n}}$ gives a system of equations,

$$
\left\{\begin{aligned}
x_{n} x_{n-1} & =\frac{y_{n}+z_{n}}{y_{n}^{2}-\frac{\alpha^{2}}{4}} \\
y_{n}+y_{n+1} & =\frac{1}{x_{n}}\left(\frac{t}{\sqrt{2}}-\frac{1}{x_{n}}\right)
\end{aligned}\right.
$$

with $z_{n}=n+\alpha / 2$. The initial conditions are given by $a_{0}=0, b_{0}=\mu_{1} / \mu_{0}$ or

$$
y_{0}=-\frac{\alpha}{2}, \quad x_{0}=\frac{\sqrt{2} \mu_{0}}{t \mu_{0}-2 \mu_{1}} .
$$

This proves Theorem 1.1 
2.3. Relation with $\mathbf{d P}_{\mathbf{I}}$. The recurrence coefficients of orthogonal polynomials for the Freud weight

$$
w_{\alpha}(x)=|x|^{2 \alpha+1} \exp \left(-x^{4}+t x^{2}\right), \quad x \in \mathbb{R}, \alpha>-1,
$$

were studied by Freud [5] (see also [11], 14]). The set $\left\{q_{n}\right\}$ of orthonormal polynomials with respect to $w_{\alpha}$ satisfies the recurrence relation

$$
x q_{n}(x)=A_{n+1} q_{n+1}(x)+A_{n} q_{n-1}(x),
$$

and the recurrence coefficients satisfy

$$
4 A_{n}^{2}\left(A_{n-1}^{2}+A_{n}^{2}+A_{n+1}^{2}-t / 2\right)=n+(2 \alpha+1) \Delta_{n},
$$

where $\Delta_{n}=0$ when $n$ is even and $\Delta_{n}=1$ when $n$ is odd. This equation is an instance of the discrete Painlevé equation $\mathrm{dP}_{\mathrm{I}}$.

Now consider the semiclassical Laguerre weight from Theorem 1.1 .

$$
v_{\alpha}(x)=x^{\alpha} \exp \left(-x^{2}+t x\right), \quad x \in \mathbb{R}^{+}, \alpha>-1,
$$

and the set $\left\{p_{n}^{\alpha}\right\}$ of orthonormal polynomials for this weight. We will denote the recurrence relation for this weight by

$$
x p_{n}^{\alpha}(x)=a_{n+1}^{\alpha} p_{n+1}^{\alpha}(x)+b_{n}^{\alpha} p_{n}^{\alpha}(x)+a_{n}^{\alpha} p_{n-1}^{\alpha}(x) .
$$

As was shown in [3], the polynomials

$$
q_{2 n}(x)=p_{n}^{\alpha}\left(x^{2}\right), \quad q_{2 n+1}(x)=x p_{n}^{\alpha+1}\left(x^{2}\right)
$$

are orthonormal with respect to the weight $w_{\alpha}$ and the following relations hold between the recurrence coefficients of both weights:

$$
\left\{\begin{array} { l } 
{ a _ { n } ^ { \alpha } = A _ { 2 n } A _ { 2 n - 1 } , } \\
{ b _ { n } ^ { \alpha } = A _ { 2 n } ^ { 2 } + A _ { 2 n + 1 } ^ { 2 } , }
\end{array} \quad \left\{\begin{array}{l}
a_{n}^{\alpha+1}=A_{2 n} A_{2 n+1}, \\
b_{n}^{\alpha+1}=A_{2 n+2}^{2}+A_{2 n+1}^{2} .
\end{array}\right.\right.
$$

Adding the equations

$$
\left\{\begin{array}{l}
4 A_{2 n}^{2}\left(A_{2 n-1}^{2}+A_{2 n}^{2}+A_{2 n+1}^{2}\right)=2 n+2 t A_{2 n}^{2} \\
4 A_{2 n+1}^{2}\left(A_{2 n}^{2}+A_{2 n+1}^{2}+A_{2 n+2}^{2}\right)=2 n+2 \alpha+2+2 t A_{2 n+1}^{2}
\end{array}\right.
$$

gives

$$
4\left(a_{n}^{\alpha}\right)^{2}+4\left(a_{n+1}^{\alpha}\right)^{2}+4\left(b_{n}^{\alpha}\right)^{2}=4 n+2 \alpha+2+2 t b_{n}^{\alpha},
$$

which is exactly the second equation of (2.10). To obtain the first equation of (2.10) one multiplies the equations

$$
\left\{\begin{array}{l}
4 A_{2 n}^{2}\left(A_{2 n}^{2}+A_{2 n+1}^{2}-t / 2\right)=2 n-4 A_{2 n}^{2} A_{2 n-1}^{2} \\
4 A_{2 n-1}^{2}\left(A_{2 n-1}^{2}+A_{2 n-2}^{2}-t / 2\right)=2 n+2 \alpha-4 A_{2 n}^{2} A_{2 n-1}^{2} .
\end{array}\right.
$$

In this way the transformations in (2.12) can be considered as a Miura transformation relating $\mathrm{dP}_{\mathrm{I}}$ (2.11) and the asymmetric $\mathrm{dP}_{\mathrm{IV}}$ (1.1) or (2.10), as described in [13. 
2.4. Orthogonality on the real line. So far we have considered the weight $w(x)=x^{\alpha} e^{-x^{2}+t x}$ on the positive real axis $\mathbb{R}^{+}$. On the full real axis we may consider the weight

$$
\tilde{w}(x)=|x|^{\alpha} e^{-x^{2}+t x}, \quad x \in \mathbb{R} .
$$

Observe that it satisfies the same Pearson equation

$$
[x \tilde{w}(x)]^{\prime}=\left(-2 x^{2}+t x+\alpha+1\right) \tilde{w}(x) .
$$

All the computations remain valid, and the conclusion is that the recurrence coefficients for the orthogonal polynomials satisfy the same discrete Painlevé equation (1.1) but with a different initial value for $b_{0}$ :

$$
a_{0}=0, \quad b_{0}=\frac{\int_{-\infty}^{\infty} x|x|^{\alpha} e^{-x^{2}+t x} d x}{\int_{-\infty}^{\infty}|x|^{\alpha} e^{-x^{2}+t x} d x} .
$$

The $b_{n}$ need no longer all be positive. The special case $t=0$ gives a symmetric weight on the real line, and hence $b_{n}=0$ for all $n \geq 0$. If we use this information in (1.1), then $1 / x_{n}=0$ for all $n \geq 0$ and (1.1) reduces to

$$
\left\{\begin{array}{l}
y_{n}^{2}=\alpha^{2} / 4 \\
y_{n}+y_{n+1}=0
\end{array}\right.
$$

With the initial value $y_{0}=-\alpha / 2$, we then find that $y_{n}=(-1)^{n+1} \alpha / 2$, so that $2 a_{n}^{2}=n+\alpha \Delta_{n}$, where $\Delta_{n}=0$ when $n$ is even and $\Delta_{n}=1$ when $n$ is odd. These are indeed the recurrence coefficients for the generalized Hermite polynomials, given in Chihara [3, Ch. V, Eq. (2.46)].

Even more is true. We may consider the weights

$$
\hat{w}(x)= \begin{cases}c_{1} x^{\alpha} e^{-x^{2}+t x}, & x>0, \\ c_{2}(-x)^{\alpha} e^{-x^{2}+t x}, & x<0,\end{cases}
$$

with $c_{1}, c_{2}>0$. They still satisfy the same Pearson equation, and all the calculus done before remains valid. Hence the recurrence coefficients for the orthogonal polynomials with weight $\hat{w}$ also satisfy (1.1) but with initial conditions

$$
a_{0}=0, \quad b_{0}=\frac{c_{1} \int_{0}^{\infty} x^{\alpha+1} e^{-x^{2}+t x} d x+c_{2} \int_{-\infty}^{0} x|x|^{\alpha} e^{-x^{2}+t x} d x}{c_{1} \int_{0}^{\infty} x^{\alpha} e^{-x^{2}+t x} d x+c_{2} \int_{-\infty}^{0}|x|^{\alpha} e^{-x^{2}+t x} d x} .
$$

The case $c_{2}=0$ gives the weight $w$ on the positive real line; the case $c_{1}=c_{2}$ gives the weight $\tilde{w}$ on the full real line. If we define $\beta=c_{1} / c_{2}$, then this gives a one-parameter family of solutions of (1.1) with $a_{0}=0$ and

$$
b_{0}=b_{0}(\beta)=\frac{\beta \int_{0}^{\infty} x^{\alpha+1} e^{-x^{2}+t x} d x+\int_{-\infty}^{0} x|x|^{\alpha} e^{-x^{2}+t x} d x}{\beta \int_{0}^{\infty} x^{\alpha} e^{-x^{2}+t x} d x+\int_{-\infty}^{0}|x|^{\alpha} e^{-x^{2}+t x} d x},
$$

where $\beta \in(0, \infty)$. Observe that $b_{0}(\beta)$ is an increasing function of $\beta$. The limiting cases $\beta=0$ and $\beta=\infty$ correspond to orthogonal polynomials on $\mathbb{R}^{-}$and $\mathbb{R}^{+}$ respectively. Hence for $b_{0}(0) \leq b_{0} \leq b_{0}(\infty)$ the solution of (1.1) corresponds to recurrence coefficients of orthogonal polynomials, and hence this solution has no singularities.

This concludes our findings in the field of orthogonal polynomials on the real line. In the next section we will look at $q$-orthogonal polynomials. 


\section{3. $q \mathrm{P}_{\mathrm{V}}$ FOR A $q$-SEMiClassical LAgUerRe WEIGHT}

In this section we investigate the weight function

$$
w(x)=\frac{x^{\alpha}}{\left(-x^{2} ; q^{2}\right)_{\infty}}
$$

on $\mathbb{R}^{+}$and the recurrence coefficients associated to the set of the monic polynomials orthogonal with respect to this weight. Here, $0<q<1, \alpha>0$ and $(z ; q)_{\infty}$ is the $q$-Pochhammer symbol

$$
(z ; q)_{\infty}=\prod_{k=0}^{\infty}\left(1-z q^{k}\right) .
$$

The weight $w$ is a semiclassical variation of a $q$-analogue of the Laguerre weight

$$
w(x)=\frac{x^{\alpha}}{(-x ; q)_{\infty}}, \quad x \in \mathbb{R}^{+},
$$

studied by Chen and Ismail in [2]. They use the theory of $q$-ladder operators to retrieve explicit expressions for the recurrence coefficients $\alpha_{n}, \beta_{n}$ of the recurrence relation

$$
x P_{n}(x)=P_{n+1}(x)+\alpha_{n} P_{n}(x)+\beta_{n} P_{n-1}(x)
$$

for the monic orthogonal polynomials $P_{n}$ associated with this weight $\left(P_{-1}=0\right)$. In the next subsection we will take a closer look at the $q$-ladder operators before applying this to the weight (3.1) to find $q \mathrm{P}_{\mathrm{V}}$ as the equation describing the recurrence coefficients of a $q$-modification of the Laguerre weight in Section 3.2

3.1. $q$-Ladder operators. The theory of ladder operators as introduced in [2] considers monic polynomials orthogonal with respect to the weight $w$ on the positive real axis. Denoting the polynomials by $\left\{P_{n}\right\}$, the orthogonality condition is

$$
\int_{0}^{\infty} P_{n}(x) P_{m}(x) w(x) d x=\zeta_{n} \delta_{m, n} .
$$

The potential $u$ is defined as

$$
u(x)=-\frac{D_{q^{-1}} w(x)}{w(x)},
$$

where $D_{q}$ is the $q$-difference operator

$$
\left(D_{q} f\right)(x)= \begin{cases}\frac{f(x)-f(q x)}{x(1-q)} & \text { if } x \neq 0, \\ f^{\prime}(0) & \text { if } x=0 .\end{cases}
$$

The main result involves two entities,

$$
\begin{aligned}
& A_{n}(x)=\frac{1}{\zeta_{n}} \int_{0}^{\infty} \frac{u(q x)-u(y)}{q x-y} P_{n}(y) P_{n}(y / q) w(y) d y \\
& B_{n}(x)=\frac{1}{\zeta_{n-1}} \int_{0}^{\infty} \frac{u(q x)-u(y)}{q x-y} P_{n}(y) P_{n-1}(y / q) w(y) d y
\end{aligned}
$$

which appear in the lowering relation [2, Theorem 1.1]

$$
D_{q} P_{n}(x)=\beta_{n} A_{n} P_{n-1}(x)-B_{n} P_{n}(x) .
$$


Furthermore, these quantities $A_{n}$ and $B_{n}$ satisfy two difference relations,

$$
\begin{gathered}
B_{n+1}(x)+B_{n}(x)=\left(x-\alpha_{n}\right) A_{n}(x)+x(q-1) \sum_{j=0}^{n} A_{j}(x)-u(q x), \\
1+\left(x-\alpha_{n}\right) B_{n+1}(x)-\left(q x-\alpha_{n}\right) B_{n}(x)=\beta_{n+1} A_{n+1}(x)-\beta_{n} A_{n-1}(x) .
\end{gathered}
$$

These equations will enable us to find expressions for the recurrence coefficients $\alpha_{n}, \beta_{n}$ as solutions of $q \mathrm{P}_{\mathrm{V}}$.

3.2. Computations. Given the weight

$$
w(x)=\frac{x^{\alpha}}{\left(-x^{2} ; q^{2}\right)_{\infty}}, \quad x \in \mathbb{R}^{+},
$$

we have the Pearson equation

$$
w\left(\frac{x}{q}\right)=q^{-\alpha+2} \frac{w(x)}{q^{2}+x^{2}} .
$$

This leads to the following expression for the potential:

$$
u(x)=\frac{q}{1-q}\left(\frac{1-q^{-\alpha}}{x}+\frac{q^{-\alpha} x}{x^{2}+q^{2}}\right),
$$

so that

$$
\frac{u(q x)-u(y)}{q x-y}=\frac{1}{1-q}\left(\frac{q^{-\alpha}-1}{x y}+\frac{q^{-\alpha}(q-x y)}{\left(x^{2}+1\right)\left(y^{2}+q^{2}\right)}\right) .
$$

Before being able to give expressions for $A_{n}, B_{n}$ we introduce the following notation for the coefficients of $P_{n}$ :

$$
P_{n}(x)=x^{n}+p_{1}(n) x^{n-1}+p_{2}(n) x^{n-2}+\cdots,
$$

where the boundary conditions $p_{1}(0), p_{2}(1), p_{2}(0), \ldots$ are all zero. This enables us to write the first terms of the Fourier series of both $P_{n}(q x)$ and $x P_{n}(q x)$ :

$$
\begin{aligned}
P_{n}(q x)= & q^{n} P_{n}(x)+(1-q) q^{n-1} p_{1}(n) P_{n-1}(x) \\
& +q^{n-2}(1-q)\left[p_{2}(n)(1+q)-q p_{1}(n) p_{1}(n-1)\right] P_{n-2}(x)+\cdots, \\
x P_{n}(q x)= & q^{n} P_{n+1}(x)+q^{n-1}\left[p_{1}(n)-q p_{1}(n+1)\right] P_{n}(x) \\
& +q^{n-2}\left[p_{2}(n)-q^{2} p_{2}(n+1)-q p_{1}(n)^{2}\right. \\
& \left.\quad+q^{2} p_{1}(n) p_{1}(n+1)\right] P_{n-1}(x)+\cdots .
\end{aligned}
$$

Note that we can express the recurrence coefficients in terms of the coefficients of the polynomials as

$$
\begin{aligned}
\alpha_{n} & =p_{1}(n)-p_{1}(n+1), \\
\beta_{n} & =p_{2}(n)-p_{2}(n+1)-p_{1}(n)^{2}+p_{1}(n) p_{1}(n+1) .
\end{aligned}
$$

We then get

$$
\begin{aligned}
A_{n}(x) & =\frac{1}{\zeta_{n}} \frac{q^{-\alpha}-1}{1-q} \frac{1}{x} \int_{0}^{\infty} P_{n}(y) P_{n}(y / q) \frac{w(y)}{y} d y \\
& +\frac{1}{\zeta_{n}} \frac{q^{-\alpha}}{1-q} \frac{1}{1+x^{2}} \int_{0}^{\infty}(q-x y) P_{n}(y) P_{n}(y / q) \frac{w(y)}{y^{2}+q^{2}} d y .
\end{aligned}
$$

Denoting

$$
R_{n}=\frac{1}{\zeta_{n}} \frac{q^{-1}-1}{1-q} \int_{0}^{\infty} P_{n}(y) P_{n}(y / q) \frac{w(y)}{y} d y
$$


we get

$$
A_{n}(x)=\frac{R_{n}}{x}+\frac{1}{\zeta_{n}} \frac{q^{-2}}{1-q} \frac{1}{1+x^{2}} \int_{0}^{\infty}(q-x y) P_{n}(y) P_{n}(y / q) w(y / q) d y
$$

Using the substitution $v=y / q$ this gives

$$
\begin{aligned}
A_{n}(x) & =\frac{R_{n}}{x}+\frac{1}{\zeta_{n}} \frac{1}{1-q} \frac{1}{1+x^{2}} \int_{0}^{\infty}(1-x v) P_{n}(q v) P_{n}(v) w(v) d v \\
& =\frac{R_{n}}{x}+\frac{q^{n}}{1-q} \frac{1}{1+x^{2}}-\frac{q^{n-1}}{1-q} \frac{x}{1+x^{2}}\left[p_{1}(n)-q p_{1}(n+1)\right]
\end{aligned}
$$

Introducing $D_{n}=p_{1}(n)-q p_{1}(n+1)$ we finally arrive at

$$
A_{n}(x)=\frac{R_{n}}{x} \frac{q^{n}}{1-q} \frac{1}{1+x^{2}}-\frac{q^{n-1} D_{n}}{1-q} \frac{x}{1+x^{2}} .
$$

Note that

$$
\sum_{j=0}^{n} q^{j-1} D_{j}=-q^{n} p_{1}(n+1)
$$

For $B_{n}$ we get

$$
\begin{aligned}
B_{n}(x) & =\frac{1}{\zeta_{n-1}} \frac{q^{-\alpha}-1}{1-q} \frac{1}{x} \int_{0}^{\infty} P_{n}(y) P_{n-1}(y / q) \frac{w(y)}{y} d y \\
& +\frac{1}{\zeta_{n-1}} \frac{q^{-\alpha}}{1-q} \frac{1}{1+x^{2}} \int_{0}^{\infty}(q-x y) P_{n}(y) P_{n-1}(y / q) \frac{w(y)}{q^{2}+y^{2}} d y
\end{aligned}
$$

Denoting

$$
r_{n}=\frac{1}{\zeta_{n-1}} \frac{q^{-\alpha}-1}{1-q} \int_{0}^{\infty} P_{n}(y) P_{n-1}(y / q) \frac{w(y)}{y} d y
$$

and using manipulations similar to those in the calculation of $A_{n}$, we get

$$
\begin{aligned}
B_{n}(x)= & \frac{r_{n}}{x}+\frac{q^{n-1} p_{1}(n)}{1+x^{2}} \\
& -\frac{q^{n-2}}{1-q} \frac{x}{1+x^{2}}\left[p_{2}(n)-q^{2} p_{2}(n+1)-q p_{1}(n)^{2}+q^{2} p_{1}(n) p_{1}(n+1)\right] .
\end{aligned}
$$

Introducing

$$
C_{n}=q^{n-2}\left(p_{2}(n)-q^{2} p_{2}(n+1)-q p_{1}(n)^{2}+q^{2} p_{1}(n) p_{1}(n+1)\right)
$$

we arrive at

$$
B_{n}(x)=\frac{r_{n}}{x}+\frac{q^{n-1} p_{1}(n)}{1+x^{2}}-\frac{1}{1-q} \frac{x}{1+x^{2}} C_{n}
$$

Inserting these expressions for $A_{n}$ and $B_{n}$ in (3.2) and (3.3) and multiplying by $x\left(1+x^{2}\right)$ gives two polynomial identities. Comparing coefficients of powers of $x$ 
gives, for (3.2), the following identities:

$$
\begin{aligned}
& r_{n+1}+r_{n}-\frac{C_{n+1}}{1-q}-\frac{C_{n}}{1-q}=-\alpha_{n} R_{n}+\frac{q^{n}}{1-q}+\alpha_{n} D_{n} \frac{q^{n-1}}{1-q}-\frac{1-q^{n+1}}{1-q}-\frac{1}{1-q}, \\
& \text { (3.7) } \quad 0=R_{n}-\frac{q^{n-1}}{1-q} D_{n}-(1-q) \sum_{j=0}^{n} R_{j}-q^{n} p_{1}(n+1) .
\end{aligned}
$$

We can simplify (3.6) by subtracting (3.4), which gives

$$
-C_{n+1}-C_{n}=q^{n}+\alpha_{n} q^{n-1} D_{n}-1+q^{n+1}-q^{-\alpha} .
$$

From (3.3) we get the identities

$$
\begin{aligned}
& q^{n} p_{1}(n+1)+\alpha_{n} \frac{C_{n+1}}{1-q}-q^{n} p_{1}(n)-\alpha_{n} \frac{C_{n}}{1-q}=-\beta_{n+1} \frac{q^{n}}{1-q} D_{n+1}+\beta_{n} \frac{q^{n-2}}{1-q} D_{n-1}, \\
& 1+r_{n+1}-\frac{1}{1-q} C_{n+1}-q r_{n}+\frac{q}{1-q} C_{n}=0,
\end{aligned}
$$

where we have already simplified (3.11) by using (3.9). We will now express $D_{n}$ in terms of $R_{n}$. First we rewrite (3.7) as

$$
0=q R_{n}-\frac{q^{n-1}}{1-q} D_{n}-(1-q) \sum_{j=0}^{n-1} R_{j}-q^{n} p_{1}(n+1) .
$$

We can now substitute $\sum R_{j}$ by using (3.7) for $n-1$. This results in the equation

$$
0=q R_{n}-R_{n-1}-\frac{q^{n-1}}{1-q} D_{n}+\frac{q^{n-2}}{1-q} D_{n-1}-q^{n} p_{1}(n+1)+q^{n-1} p_{1}(n),
$$

which has integrating factor $q^{n-1}$. After taking a telescopic sum we have

$$
0=(1-q) q^{n} R_{n}-(1-q) R_{0}+q^{2 n} p_{1}(n+1)-q^{2 n-1} p_{1}(n)+q^{-1} p_{1}(0) .
$$

However, all terms with $R_{0}, p_{1}(0)$ and $p_{1}(1)$ cancel each other when we consider (3.7) for $n=0$. After dividing by $q^{n}$ we are left with the identity

$$
(1-q) R_{n}=q^{n-1}\left(p_{1}(n)-q p_{1}(n+1)\right)=q^{n-1} D_{n} .
$$

Next, we write $C_{n}$ in terms of $r_{n}$. We use (3.11) to obtain

$$
\alpha_{n} q^{n}+\frac{\alpha_{n}}{1-q} C_{n+1}-\frac{\alpha_{n}}{1-q} C_{n}=\beta_{n+1} R_{n+1}-\beta_{n} R_{n-1}
$$

together with (3.9) to obtain, after dividing by $\alpha_{n}$,

$$
r_{n+1}-r_{n}=-q^{n}+\frac{1}{1-q} C_{n+1}-\frac{1}{1-q} C_{n} .
$$


Taking a telescopic sum and using $r_{0}=C_{0}=0$, we find that

$$
(1-q) r_{n}=C_{n}-1+q^{n} .
$$

We now use (3.4) to substitute $\alpha_{n} R_{n}$ in (3.10):

$$
q r_{n+1}-r_{n}+q^{-\alpha}=\beta_{n+1} \frac{q^{n+1}}{1-q}-\beta_{n} \frac{q^{n-1}}{1-q} .
$$

This expression has integrating factor $q^{n}$. Taking a telescopic sum, we get

$$
\beta_{n} \frac{q^{n-1}}{1-q}=r_{n}+q^{-\alpha-n} \frac{1-q^{n}}{1-q}
$$

We have now been able to write $\beta_{n}, C_{n}$ and $D_{n}$ in terms of $r_{n}$ and $R_{n}$. The same can be done for $\alpha_{n}$. Combining (3.5) and (3.7) results in

$$
q^{n-1} p_{1}(n)=R_{n}-\alpha_{n} \frac{q^{n}}{1-q} \text {. }
$$

We would like to get rid of the $p_{1}(n)$ in this equation. For that purpose, we rewrite (3.9) using (3.14) and (3.13) to find that

$\alpha_{n}(1-q) q^{-\alpha-n-1}=\beta_{n+1} q^{n}\left(p_{1}(n)-q p_{1}(n+2)\right)-\beta_{n} q^{n-2}\left(p_{1}(n-1)-q p_{1}(n+1)\right)$.

This equation has an integrating factor $q^{n+1}$, and after taking a telescopic sum we get

$$
\begin{aligned}
q^{-\alpha}(1-q) p_{1}(n) & =\beta_{n} q^{n-1}\left(q p_{1}(n+1)-p_{1}(n-1)\right) \\
& =\beta_{n} q^{n-1}\left(-q \alpha_{n}-q^{-n+2}(1-q) R_{n-1}\right) .
\end{aligned}
$$

Combining (3.15) and (3.16) we get

$$
\alpha_{n}\left(q^{n}-q^{3 n+\alpha-1} \beta_{n}\right)=(1-q) R_{n}+(1-q) R_{n-1} q^{2 n+\alpha} \beta_{n},
$$

which expresses $\alpha_{n}$ as a function of $R_{n}$ and $r_{n}$.

If we look at (3.9), we see that the right hand side has an integrating factor $R_{n}$. When multiplying (3.9) with this integrating factor, the expression $\alpha_{n} R_{n}$ appears on its left hand side, and we use (3.4) to substitute it in terms of $r_{n}$, which gives

$$
\beta_{n+1} R_{n+1} R_{n}-\beta_{n} R_{n} R_{n-1}=r_{n+1}^{2}-r_{n}^{2}+\frac{1-q^{-\alpha}}{1-q}\left(r_{n+1}-r_{n}\right) .
$$

Taking a telescopic sum gives

$$
\beta_{n} R_{n} R_{n-1}=r_{n}\left(r_{n}+\frac{1-q^{-\alpha}}{1-q}\right) .
$$

It is this equation we will use, together with (3.4), to recover $q \mathrm{P}_{\mathrm{V}}$. First, we rewrite (3.4) using the expression found for $\alpha_{n}$ :

$q^{n}\left(r_{n+1}+r_{n}+\frac{1-q^{-\alpha}}{1-q}\right)\left(q^{2 n+\alpha-1} \beta_{n}-1\right)=(1-q) R_{n}^{2}+(1-q) q^{2 n+\alpha} \beta_{n} R_{n} R_{n-1}$.

Using (3.17) in this equation gives an expression for $R_{n}^{2}$ :

$$
\begin{aligned}
(1-q) R_{n}^{2}= & q^{n}\left(r_{n+1}+r_{n}+\frac{1-q^{-\alpha}}{1-q}\right)\left(q^{2 n+\alpha-1} \beta_{n}-1\right) \\
& -(1-q) q^{2 n+\alpha} r_{n}\left(r_{n}+\frac{1-q^{-\alpha}}{1-q}\right) .
\end{aligned}
$$


We insert this in the squared (3.17),

$$
\beta_{n}^{2} R_{n}^{2} R_{n-1}^{2}=r_{n}^{2}\left(r_{n}+\frac{1-q^{-\alpha}}{1-q}\right)^{2},
$$

and replace all $\beta_{n}$ using (3.14). This gives a difference equation for $r_{n}$ :

$$
\begin{aligned}
&(1-q)^{2} r_{n}^{2}\left(r_{n}+\right.\left.\frac{1-q^{-\alpha}}{1-q}\right)^{2}=q\left((1-q) r_{n}+\left(1-q^{n} q^{-n-\alpha}\right)\right)^{2} \\
& \times\left\{\left(r_{n+1}+r_{n}+\frac{1-q^{-\alpha}}{1-q}\right)\left((1-q) q^{n+\alpha} r_{n}-q^{n}\right)\right. \\
&\left.-(1-q) q^{n+\alpha} r_{n}\left(r_{n}+\frac{1-q^{-\alpha}}{1-q}\right)\right\} \\
& \times\left\{\left(r_{n-1}+r_{n}+\frac{1-q^{-\alpha}}{1-q}\right)\left((1-q) q^{n+\alpha-1} r_{n-1}-q^{n-1}\right)\right. \\
&\left.-(1-q) q^{n+\alpha-1} r_{n-1}\left(r_{n-1}+\frac{1-q^{-\alpha}}{1-q}\right)\right\} .
\end{aligned}
$$

We now use the substitution $x_{n}=q^{-\alpha / 2}-q^{\alpha / 2}(1-q) r_{n}=q^{-n-\alpha / 2}-q^{n+\alpha / 2-1} \beta_{n}$ and obtain

$$
\left(x_{n} x_{n+1}-1\right)\left(x_{n} x_{n-1}-1\right)=\frac{q^{-2 n-\alpha}\left(x_{n}-q^{-\alpha / 2}\right)^{2}\left(x_{n}-q^{\alpha / 2}\right)^{2}}{\left(x_{n}-q^{-n-\alpha / 2}\right)^{2}},
$$

which is $q \mathrm{P}_{\mathrm{V}}$. Expressed in terms of the $k$ th moments $\mu_{k}$ of the weight $w$, the initial values are

$$
x_{0}=q^{-\alpha / 2}, \quad x_{1}=q^{-1-\alpha / 2}-q^{\alpha / 2} \frac{\mu_{2} \mu_{0}-\mu_{1}^{2}}{\mu_{0}^{2}} .
$$

This concludes the proof of Theorem 1.3 .

\section{Conclusions and outlook}

In this paper we have established the connection between various semiclassical variations on the Laguerre weight and discrete Painlevé equations for the recurrence coefficients of the corresponding orthogonal polynomials.

In the case of semiclassical Laguerre orthogonal polynomials (on the positive half-line) we found a connection to the system (1.2) which reduces to $\mathrm{dP}_{\mathrm{IV}}$ in a special case. We recovered an earlier result about the quadratic relation between $\mathrm{dP}_{\mathrm{IV}}$ and $\mathrm{dP}_{\mathrm{I}}$ from the orthogonal polynomial point of view.

The case of the semiclassical $q$-Laguerre orthogonal polynomials gives a connection with $q \mathrm{P}_{\mathrm{V}}$. As we know from the coalescence cascades for discrete Painlevé equations, $\mathrm{dP}_{\mathrm{IV}}$ is a limiting case of $q \mathrm{P}_{\mathrm{V}}$, just like the semiclassical Laguerre weight on the positive half-line can be found from the semiclassical $q$-Laguerre weight in an appropriate limit. The symmetrized weight

$$
w(x)=\frac{|x|^{2 \alpha+1}}{\left(-x^{4} ; q^{4}\right)_{\infty}},
$$

on the full real line was studied by Ismail and Mansour [10. For $\alpha=-1 / 2$ they found a $q$-discrete form of $\mathrm{P}_{34}$ (see [7, Eq. (6.3)]). One would expect $q \mathrm{P}_{\text {III }}$ because there is a known quadratic Miura transformation between $q \mathrm{P}_{\mathrm{V}}$ and $q \mathrm{P}_{\mathrm{III}}$. We believe that this could be studied in more detail. 


\section{REFERENCES}

[1] M. P. Bellon, C.-M. Viallet, Algebraic entropy, Commun. Math. Phys. 204 (1999), 425-437. MR:1704282 (2000f:37040)

[2] Y. Chen, M. Ismail, Ladder operators for q-orthogonal polynomials, J. Math. Anal. Appl. 345 (2008), 1-10. MR2422628 (2009d:33021)

[3] T.S. Chihara, An Introduction to Orthogonal Polynomials, Mathematics and its Applications, 13, Gordon and Breach, New York, 1978. MR0481884 (58:1979)

[4] A.S. Fokas, A.R. Its, A.V. Kitaev, Discrete Painlevé equations and their appearance in quantum gravity, Comm. Math. Phys. 142 (1991), 313-344. MR1137067 (93a:58080)

[5] G. Freud, On the coefficients in the recursion formulae of orthogonal polynomials, Proc. Roy. Irish Acad. Sect. A 76(1) (1976), 1-6. MR0419895 (54:7913)

[6] B. Grammaticos, J. Hietarinta, A. Ramani, Discrete versions of the Painlevé equations, Phys. Rev. Lett. 67 (1991), 1829-1832. MR 1125951 (92j:39011)

[7] B. Grammaticos, A. Ramani, Discrete Painlevé equations: Coalescences, limits and degeneracies, Physica A 228 (1996), 160-171. MR1399286 (97e:58131)

[8] B. Grammaticos, A. Ramani, Discrete Painlevé equations: A review, Lect. Notes Phys., 644, Springer, 2004, pp. 245-321. MR2087743 (2005g:39032)

[9] B. Grammaticos, A. Ramani, V. Papageorgiou, Do integrable mappings have the Painlevé property?, Phys. Rev. Lett. 67 (1991), 1825-1828. MR.1125950 (92f:58081)

[10] M. E. H. Ismail, Z. Mansour, q-Analogues of Freud weights and nonlinear difference equations, manuscript.

[11] A.P. Magnus, Freud's equations for orthogonal polynomials as discrete Painlevé equations, in Symmetries and Integrability of Difference Equations (Canterbury, 1996), London Math. Soc. Lecture Note Ser., 255, Cambridge University Press, 1999, pp. 228-243. MR1705232 (2000k:42036)

[12] F. Nijhoff, On a q-deformation of the discrete Painlevé I equation and q-orthogonal polynomials, Lett. Math. Phys. 30 (1994), 327-336. MR.1271093 (95e:33024)

[13] A. Ramani, B. Grammaticos, T. Tamizhmani, Quadratic relations in continuous and discrete Painlevé equations, J. Phys. A. 33 (2000), 3033-3044. MR.1766506 (2001d:34018)

[14] W. Van Assche, Discrete Painlevé equations for recurrence coefficients of orthogonal polynomials, in "Difference Equations, Special Functions and Orthogonal Polynomials" (S. Elaydi et al., eds.), World Scientific, 2007, pp. 687-725. MR.2451211(2009k:42055)

Department of Mathematics, Katholieke Universiteit Leuven, Celestijnenlaan 200B, BE-3001 Leuven, Belgium

E-mail address: lies.boelen@wis.kuleuven.be

Department of Mathematics, Katholieke Universiteit Leuven, Celestijnenlaan 200B, BE-3001 LEuven, BELGium

E-mail address: walter@wis.kuleuven.be 